\title{
Using tripartite group area as a measure of social interactions in pre-school children: A pilot study
}

\author{
Masashi Tsukamoto $^{1,2}$ (D) $\cdot$ Airi Tsuji ${ }^{3,4} \cdot$ Satoru Sekine ${ }^{5,6} \cdot$ Takahide Omori $^{7} \cdot$ Kenji Suzuki $^{3} \cdot$ Junichi Yamamoto $^{7}$
}

Accepted: 22 March 2021 / Published online: 26 April 2021

(C) The Author(s) 2021

\begin{abstract}
This study aimed to measure tripartite group area using motion capture systems and investigated whether group area could be used as a measure of pre-school children's social interactions. In Experiment 1, two typically developing girls and an adult staff member engaged in free play. In Experiment 2, two typically developing boys and two adult staff members played balloon volleyball. Both experiments had three types of measures: subjective evaluation of whether participants played together, social behaviours (e.g. eye contact for Experiment 1 and balloon tosses for Experiment 2) and group area. Results showed that group area was significantly and negatively related to subjective evaluation in Experiment 2, whereas we observed no relationship between subjective evaluation and group area in Experiment 1. Overall, however, only a low correlation was observed between subjective evaluation and group area in Experiment 2. Furthermore, there were strong sequential associations between subjective evaluation and social behaviour, rather than between subjective evaluation and group area. Although group area as an index of social interactions is less accurate than behavioural data directly observed by humans, it may be worth using as a low-cost preliminary measure, since it can be automatically calculated using motion capture systems.
\end{abstract}

Keywords Social interaction · Group area $\cdot$ Motion capture system $\cdot$ Time-window sequential analysis $\cdot$ Pre-school children

Measuring social interactions of young children is quintessential for assessing social development. In the early stages of development, joint attention with adults (e.g. parents) is an important milestone (Bakeman \& Adamson, 1984; Mundy

Masashi Tsukamoto

10mp011@stu.meisei-u.ac.jp

$\triangle$ Airi Tsuji

tsuji@ai.iit.tsukuba.ac.jp

1 Present address: Department of Psychology, School of Human Sciences, Senshu University, Kanagawa, Japan

2 Keio Advanced Research Centers, Keio University, Bunkyo City, Tokyo, Japan

3 Present address: Institute of Engineering, Tokyo University of Agriculture and Technology, Tokyo, Japan

4 Faculty of Engineering, Information and Systems, University of Tsukuba, Ibaraki, Japan

5 Present address: Department of Psychology, Keio University and Japan Society for the Promotion of Science, Tokyo, Japan

6 Graduate School of Information Science and Technology, The University of Tokyo, Bunkyo City, Tokyo, Japan

7 Department of Psychology, Keio University, Tokyo, Japan
\& Gomes, 1998), and playing with peers eventually becomes crucial to social development (Barbu et al., 2011; Howes \& Matheson, 1992). Observation is the most common approach to assessing social development in young children (Brassard \& Boehm, 2007; Neisworth \& Bagnato, 2004). For example, Autism Diagnostic Observation Schedule-2nd edition (ADOS-2; Lord et al., 2012) which is activity-based assessment that allows to observe behaviours associated with autism spectrum disorder (ASD) in standard context is often used for diagnosing ASD, characterised by persistent deficits in social communication and social interaction (APA, 2013).

Observation by multiple observers in a range of situations allows the construction of a comprehensive understanding of children's behaviour and produces information that can help the best intervention to be chosen, if intervention is necessary. There are two major observation methods, namely, direct and indirect observation. With direct observation, observers record occurrences of precisely defined behaviours in close proximity to children who exhibit the target behaviours (Miltenberger, 2017). For example, using an observationbased assessment system, Booren et al. (2012) observed variations in pre-school children's interactions and teachers' behaviours across classroom activities as well as some limited gender differences. Direct observation is also used in 
interventions based on applied behaviour analysis for children with ASD who have deficits in social interaction. For example, Vernon et al. (2012) observed and recorded children's eye contact, verbal initiation, positive affect, and synchronous engagement as measures of interaction between 2- and 4-yearold children with ASD and their parents. Indirect observation collects information on children from people familiar with them (e.g. parents and teachers) by using checklists, rating scales, questionnaires and interviews (Brassard \& Boehm, 2007). For example, the Penn Interactive Peer Play Scale assesses whether pre-school children have exhibited particular social interactions (e.g. 'shares toys with others', or 'shows positive emotion during play [e.g. smiles, laughs]') during free play in the past two months (Fantuzzo et al., 1998a; Fantuzzo et al., 1998b). Because this 4-point Likert scale (and other rating scales) consists of one version for use by the parent at home and another version for use by the teacher at pre-school or kindergarten, it is superior to direct observation for evaluating social interactions in different settings over reduced time periods with less effort, and it prevents invasions of privacy.

Although they are well established, methods of observation and related measures exhibit certain limitations in terms of their cost and accuracy (Brassard \& Boehm, 2007; Yoder et al., 2018). In direct observation, observers must understand what behaviours are targeted and be fully trained to record them. Moreover, to assess accuracy, both observers must use their recorded data to evaluate interobserver agreement. Furthermore, close observation can alter children's behaviours, even in the absence of intervention (Kazdin, 1979; Miltenberger, 2017). Although indirect observation does not require as much effort or time as direct observation, it produces less accurate data and does not reflect real-time interactions, because the information that untrained observers provide on children's behaviour is based on their memories.

Recently, Messinger et al. (2019) measured the social interactions of five-year-old children in free-play situation using the continuous measurement technology: radio-frequency identification system. Then, they suggested that such an automated technology can efficiently assess social interactions in classroom to measure children's location distributions and speed of movement. As computer technology has revolutionised the availability of behavioural observation (Thompson et al., 2000), the use of technologies that mitigate the limitations of existing methods can broaden the scope of assessment for social development.

In another study, Tsuji et al. (2018) measured positional information of two children with ASD and one adult staff member during free play using a motion capture system (MCS) and calculated the tripartite group area. They found that group area decreased after therapeutic interventions by the adult staff member, suggesting group area would be available to measure social interactions in a small group. Because
MCS automatically calculates group area and reflects the realtime interactions of the group, it may enable us to evaluate complicated and dynamic social interactions efficiently.

Although group area could provide a new perspective for measuring social interactions, it is still unclear whether group area could be a valid social index, as the relationship between group area and other measures has not been investigated in previous studies. A group area that consists of more than three individuals depends on the interpersonal distance among group members. Scholars have used interpersonal distance to assess personal space and have considered its adjustment as an important aspect of social interaction (Hayduk, 1983; Kennedy \& Adolphs, 2014). For example, Candini et al. (2017) reported that, from a first-person perspective, the interpersonal distance at which children are at ease with another person shortens after they have cooperatively interacted with that person. Such a pattern was observed not only in children who achieve age-appropriate developmental milestones (typically developing children) but also in those with ASD and relatively mild social impairment. Therefore, it is possible that Tsuji et al. (2018) found that therapeutic interactions shortened the interpersonal distance between two children with ASD and one adult staff member, which have produced a change in group area between the three members. However, given that group area is not the same measure as interpersonal distance, the relations between group area and other measures including social interaction must be clarified so that group area can be used as a social index. It could be predicted that distances between them and group area size would potentially decrease as their interactions increased.

In this study, we conducted two experiments with typically developing children as a pilot study. As a replication, we measured small group activities with two children and adult staff members, using the same system as Tsuji et al. (2018). The primary purpose of this study was to investigate whether we could observe relationships between group area, social behaviours and subjective evaluations from independent observers, and determine whether the group area calculated via MCS can be used as a measure that complements the limitations of direct and indirect observation. In Experiment 1, two children and one adult staff member engaged in free play, in a situation similar to Tsuji et al. (2018). In Experiment 2, another two children and two adult staff members played a balloon game that required approaching others.

\section{Experiment 1}

\section{Participants}

Two typically developing girls participated in the experiment (Child 1: 5 years 8 months and Child 2: 5 years 9 months). The two children attended the same kindergarten and had a good 
friendship. An adult staff member (assistant) also participated in the group activity. The assistant was a graduate student who had majored in applied behaviour analysis and had not previously met the children. After the experiment, all participants were remunerated at a rate of approximately $\$ 10$ an hour.

\section{Apparatus and Setting}

To measure each participant's position, we used OptiTrack Flex 3 MCS. The OptiTrack Flex 3 is an optical motion capture camera that tracks reflective markers and calculates their positions. MCS data were measured at 1/60 frames per second (60 fps), and the mean reprojection error (3D) was $0.54 \mathrm{~mm}$. Motive software was used for the analysis.
This study was conducted in a playroom at a private university in Japan (Fig. 1). The room was equipped with eight OptiTrack Flex 3 cameras and three video cameras (two home video cameras and one ceiling video camera). To prevent inaccurate measurements by MCS around the wall, we set up a railed activity circle in an elliptical shape, with a diameter of $4.00 \mathrm{~m}$ for the major axis and $3.25 \mathrm{~m}$ for the minor axis. During the experiments, all participants engaged in activities inside the activity circle while wearing a specialised cap with reflective markers, which collected data regarding head position, using MCS. The base cap is commonly used in kindergartens and elementary schools in Japan, and it is easy to wear even for children with hyperreactivity to sensory input (for more information on MCS and the specialised cap, please refer to Tsuji et al., 2016).

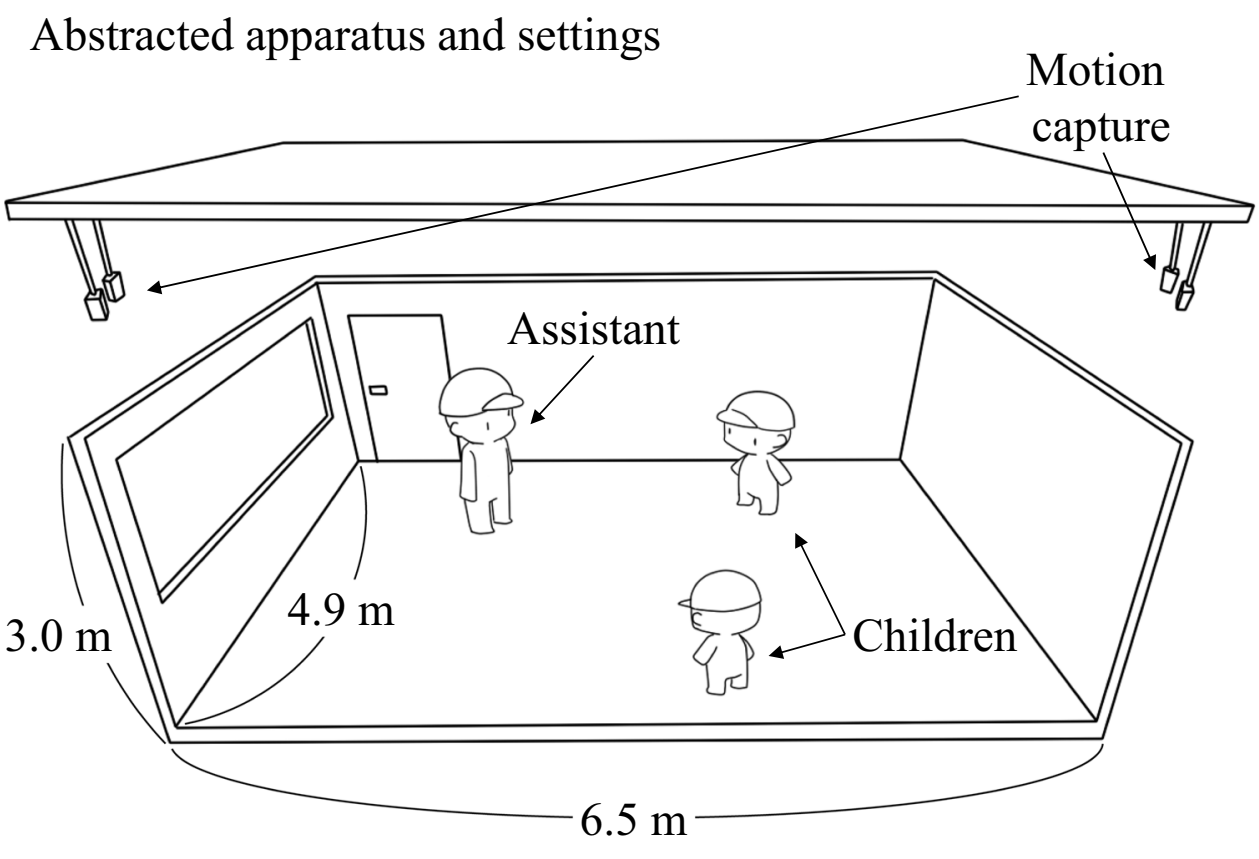

\section{A scene from the test phase in two experiments}

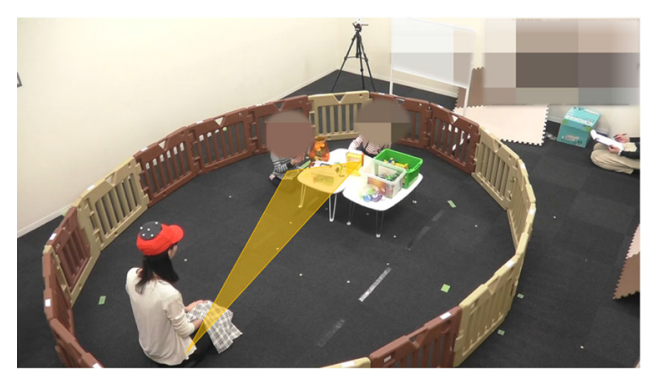

Fig. 1 Apparatus and settings. The top presents abstracted apparatus, the bottom-left presents a scene from the test phase in Experiment 1, and the bottom-right presents a scene from the test phase in Experiment 2. There were actually four pairs motion capture cameras on the four corners of the ceiling and a railed activity circle in an elliptical shape on the floor. In Experiment 1, the two children were playing with toys. The assistant did not move from a predetermined position. The yellow triangle is a

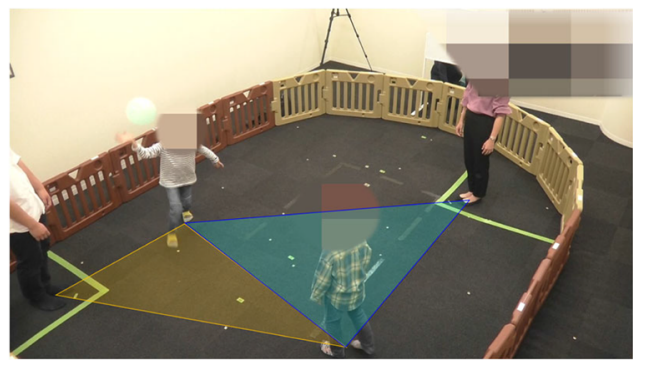

representative of tripartite group area. In Experiment 2, the two children could freely move around inside the activity circle and toss a balloon. The assistants did not move from predetermined positions. The yellow triangle is a representative of group area which consisted of both children and one assistant, and the blue triangle is a representative of group area which consisted of both children and the other assistant 


\section{Materials}

There were two low tables inside the activity circle (at the bottom-left of Fig. 1). For the test phases, we provided six types of toys familiar to the children: blocks, dinosaur dolls, Disney dolls, Pop-Up Pirate, playhouse kit and coloured pencils. For the interaction phases, three types of toys were used: a bowling kit, rubber ball and drawing kit.

\section{Experimental Design}

Experiment 1 consisted of a single session including test (test 1 , test 2 and test 3 ) and interaction phases. We assessed whether group area in the test phases changed gradually with the assistant's interaction in the interaction phases. MCS measurements and behavioural observation were performed during the test phases. Data from the interaction phases were excluded from analysis.

\section{Procedures}

Experiment 1 was carried out in the following order: test 1 , interaction 1, interaction 2, test 2, interaction 3 and test 3 (at the left side of Table 1). Each phase lasted six minutes. For preparing the next phase and rest, there were intervals of approximately two minutes between each phase. Before the experiment, the experimenter (first author) asked the children and assistant to introduce themselves to each other. The experimenter set specialised caps on the children's heads to indicate MCS measurement, telling them that they were needed to wear the cap in the playroom. An overview of the session's activities was provided. The experimenter managed the progress of the session, setting the materials before each phase, but did not interact with the children during each phase. When the children took off their caps or complained of fatigue, the experimenter encouraged them to wear the cap or let them take a short break. During the two-minute intervals between each phase, the experimenter took care of the children whereas the assistant did not interact with them.

During the test phases, six toys were set on tables for the children on one side of the activity circle, and the assistant sat on floor on the opposite side. The children played with the toys and could move freely inside the activity circle. The assistant remained in a predetermined position and took little initiative in the play, while holding her gaze towards the children and smiling (Fig. 1). When the children called her, she did not move and simply said 'I'll watch you from here'. She did not give any instructions or ask questions. When the children were clearly speaking to her, she replied naturally.

Before the beginning of test 1 , the children received the following instructions:

You and the assistant will play together using these toys. You can freely choose any toys. The assistant will be watching you from here (pointing the position). If you want to play with her, take a toy and show it to her (All references to individuals for each instruction were each person's real name. The same applies hereafter).

During the interaction phases, the children and assistant engaged in bowling, playing soccer and drawing. These activities were prepared in advance by the experimenter, based on the children's preferences and chronological age. Unlike the test phases, in the interaction phases, the assistant moved freely inside the activity circle and exhibited positive affect, such as smiling, laughing and using a playful vocal tone, to encourage the children's communicative responses.
Table 1 Summary of the session in two experiments

\begin{tabular}{|c|c|c|c|c|}
\hline \multicolumn{2}{|l|}{ Experiment 1} & \multicolumn{3}{|l|}{ Experiment 2} \\
\hline Phase & Activity & Phase & Activity & Assistant \\
\hline Test 1 & Free play & Test 1 & Ballon volleyball & $\begin{array}{l}\text { Assistant }{ }^{R F} \\
\text { Assistant }^{\text {Non-RF }}\end{array}$ \\
\hline Interaction 1 & Bowling & Interaction 1 & Bowling & Assistant $^{R F}$ \\
\hline Interaction 2 & Playing soccer & Interaction 2 & Bowling & Assistant ${ }^{\text {Non-RF }}$ \\
\hline Test 2 & Free play & Test 2 & Ballon volleyball & $\begin{array}{l}\text { Assistant }^{R F} \\
\text { Assistant }^{\text {Non-RF }}\end{array}$ \\
\hline Interaction 3 & Drawing & Interaction 3 & Playing catch & Assistant ${ }^{\text {Non-RF }}$ \\
\hline Test 3 & Free play & $\begin{array}{l}\text { Interaction } 4 \\
\text { Test } 3\end{array}$ & $\begin{array}{l}\text { Playing catch } \\
\text { Ballon volleyball }\end{array}$ & $\begin{array}{l}\text { Assistant }^{R F} \\
\text { Assistant }^{R F} \\
\text { Assistant } \\
\text { Non-RF }\end{array}$ \\
\hline
\end{tabular}

Assistant ${ }^{\mathrm{RF}}$ indicates the assistant who exhibited positive affect and intended to reinforce the child's communicative responses in the interaction phases. Assistant ${ }^{\text {Non-RF }}$ indicates the assistant who intended to provide no reinforcement in interaction phases 


\section{Measures and Dependent Variables}

Location Information with MCS During test phases, MCS kept real-time measurements and recordings of spatial positions of the participants' and assistant's heads in 3D space. After the experiment was complete, we calculated interpersonal distance and group areas using each individual's position. In Experiment 1, the tripartite group area (triangular area formed by two children and one assistant) was used for analysis. After the experiment started, we dropped a marker to the floor as a countdown and analysed data collected for six minutes, starting from the time that the marker touched the floor. In test 3 , the children took a 117-s break, as they complained of the heat. During the break, measurement was stopped. Then, the experiment was carried out until the cumulative measurement time reached six minutes.

Observational Measurement of Social Behaviours After the experiment, behavioural observation was executed by the first author using a video recorded of the three test phases. The occurrence of three social behaviours was counted: eye contact, verbal initiation and showing objects. One eye contact was coded for each time a child looked at the assistant's face. Verbal initiation was defined as a functional utterance by a child toward the assistant (e.g. "Which do you think is better, a pink one or a red one?'). A verbal initiation was coded when a child made a spontaneous utterance while facing the assistant. Answering a question, echolalia and laughter were not counted as verbal initiation. One object showing was coded when a child held an object in the sight of the assistant, while making eye contact. For the test phases, the frequency of these social behaviours for each child was used in the analysis. All behaviours were coded using Noldus Observer XT 14 software.

To evaluate the reliability of the observation, another independent observer coded all behaviours across test phases. For total counts of all three social behaviours across the three test phases, interobserver agreement (IOA) was calculated by dividing the number of smaller counts by larger counts and then multiplying the result by 100 . For Child 1 , the final IOA was $96.3 \%$ (eye contact $=95.7 \%$ [44/46], verbal initiation $=83.3 \%$ [25/30], showing objects $=100.0 \%[8 / 8]$ ). For Child 2, the final IOA was $95.0 \%$ (eye contact $=100.0 \%$ [16/16], verbal initiation $=100.0 \%[1 / 1]$, showing objects $=66.7 \%[2 / 3])$.

Subjective Evaluation of Interaction After the experiment, four independent observers who were blind to the purpose of this study watched a combined video on Observer XT 14 software and evaluated the group interactions during each test phase, using a 3 -point scale: $3=$ children are playing with the adult; $2=$ children are probably playing with the adult and $1=$ children are not playing with the adult. The observers coded the duration of each score using the three assigned keys. The observations began with a score of ' 1 ', and all activities by participants in the videos were always evaluated with one of the three possible scores. Because the scores were mutually exclusive, the observers could not code them at the same time. Before evaluation, each observer practiced recording using an unrelated video. During the evaluation, the observers watched the recording at a normal speed without rewinding and pausing. The videos were watched at random.

\section{Results}

We expected that the children's social behaviour would be enriched by their communicative experiences in the interaction phases. If this change occurred, it should be reflected in the transitions among the three types of dependent variables in the three test phases.

Tripartite Group Area The MCS measured 60 fps, and each test phase in Experiment 1 lasted six minutes; therefore, a total of 21,600 group areas should have been obtained for each phase. However, the children temporarily removed their caps, which caused deficient records. Thus, valid data were 21,600 (100\%) for test 1, 20,347 (94\%) for test 2 and 20,799 (96\%) for test 3 . We averaged the available values of group area for each second with at least one record and analysed a total of $360 \mathrm{~s}$ of data for each phase.

Fluctuations in group area are shown in Fig. 2 by a solid line. The average group area in each test phase was $0.38 \mathrm{~m}^{2}$ $(S D=0.205)$ for test $1,0.63 \mathrm{~m}^{2}(S D=0.278)$ for test 2 and $0.29 \mathrm{~m}^{2}(S D=0.129)$ for test 3 . The group area for test 3 was smaller than that for test 1 , whereas the largest group area was observed in test 2 .

Frequency of Social Behaviours The number of social behaviours is shown in Fig. 3. In test 1, the social behaviours of both children were at relatively lower levels (Child 1 : eye contact $=$ 6 , verbal initiation $=1$, showing objects $=0$; Child 2 : eye contact $=2$, verbal initiation $=0$, showing objects $=0$ ). After the interaction phases, however, Child 1 showed higher levels of social behaviours in test 2 (eye contact $=22$, verbal initiation $=16$, showing objects $=6$ ) and test 3 (eye contact $=18$, verbal initiation $=8$, showing objects $=$ 2). Although the overall number of social behaviours of Child 2 were less than those of Child 1, there was an increasing trend in eye contact (test 2 : eye contact $=6$, verbal initiation $=1$, showing objects $=0$; test 3 : eye contact $=8$, verbal initiation $=0$, showing objects $=3$ ).

Subjective Evaluation of Interaction Four observers' subjective evaluation scores were averaged for every second. Transition of average subjective evaluation is shown by a dashed line in Fig. 2. The overall mean scores were 1.00 $(S D=0.000)$ for test $1,1.01(S D=0.052)$ for test 2 and 1.01 $(S D=0.039)$ for test 3 . Almost every observer evaluated the 


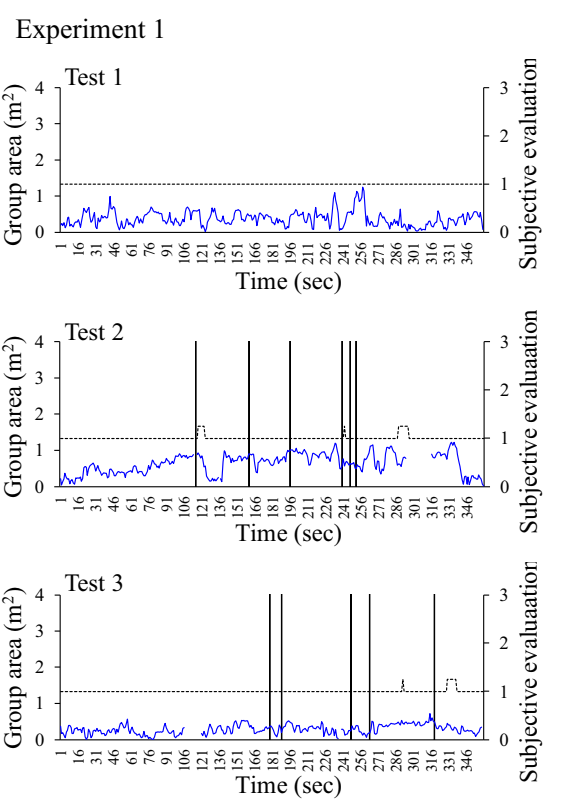

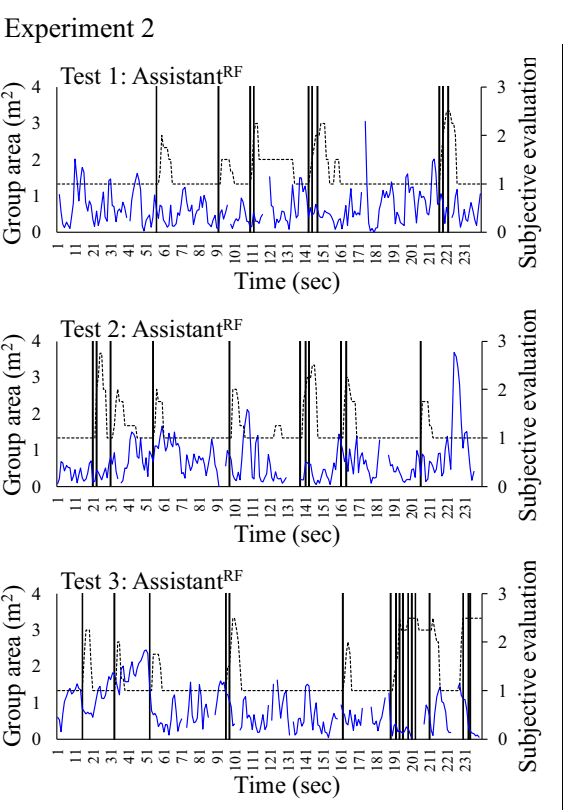
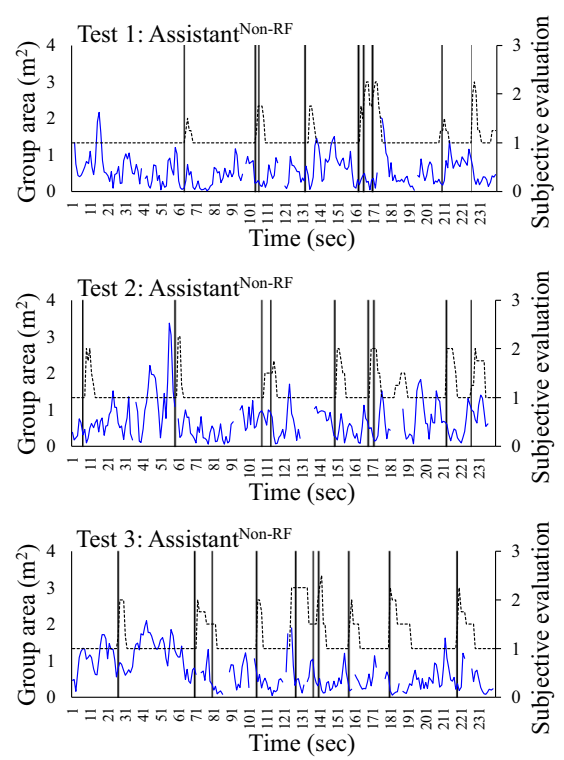

Fig. 2 Time sequential data of the group areas in test phases. The blue solid lines represent the fluctuations of group area. The dashed lines represent the transitions of average subjective evaluation. The black bars represent the children's social behaviours (showing objects for Experiment 1 and balloon tosses towards each assistant for Experiment

group interactions as ' $1=$ children are not playing with the adult' and especially in test 1 , no one rated a score of ' $2=$ they are probably playing together' or higher.

\section{Correspondence between Group Area and Subjective}

Evaluation If we could monitor the quantity of social interaction of children using the locational information by MCS, certain correspondence would be observed between fluctuations of group area and subjective evaluation score. We calculated Spearman's rank correlation coefficients between these two variables for each test phase. For the test phase 1, because no variation exists in mean subjective evaluation scores (mean scores at all seconds were 1.0), the correlation coefficient was not
2). The left represents the group areas in Experiment 1. The centre represents the group areas with the assistant who intended to reinforce the child's communicative responses in interaction phases in Experiment 2. The right represents the group areas with the assistant who intended to provide no reinforcement in interaction phases in Experiment 2

available. There were no significant correlations for the test 2 $\left(r_{s}=0.05, p=0.320\right)$ and the test $3\left(r_{s}=0.10, p=0.061\right)$.

Supplementary Episodes Overall, the children stayed close to the toys and played together. Showing objects was observed to a certain extent; however, the children never approached the assistant to show her a toy.

\section{Discussion}

In Experiment 1, we attempted to measure social interactions in a small group using MCS in a situation similar to Tsuji et al.
Fig. 3 The number of the children's social behaviours during each six-min test phase in Experiment 1. The filled bar represents eye contact, the unfilled bar represents verbal initiation and the hatched bar represents showing objects

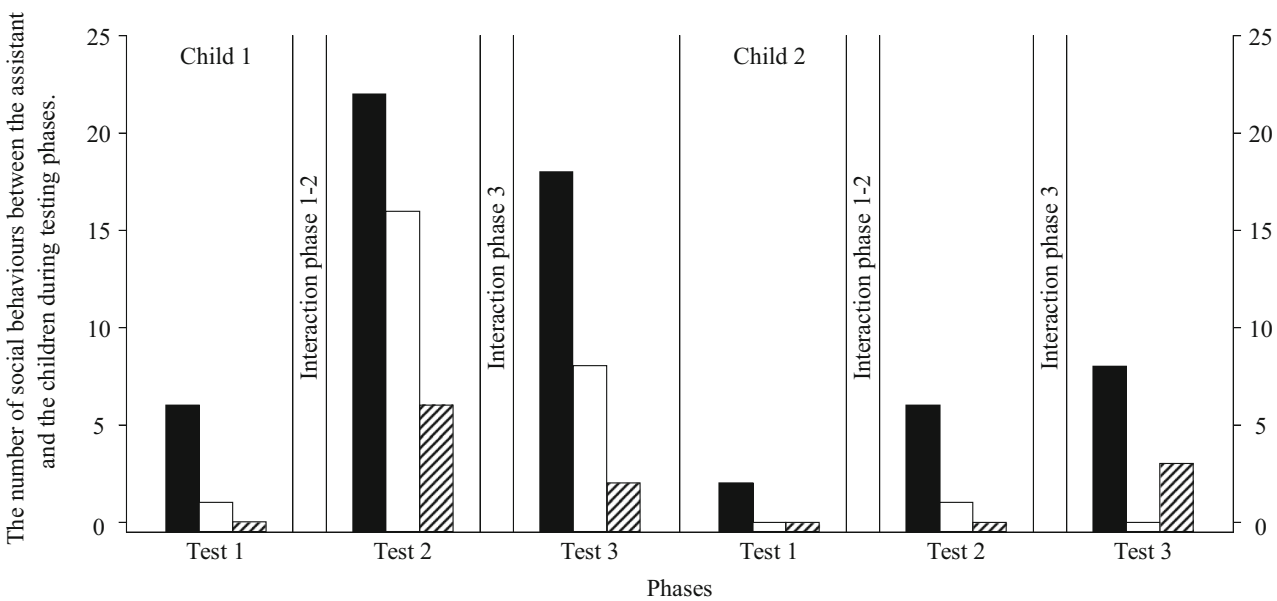


(2018). We had predicted a negative correlation between group area and subjective evaluation, because group area would possibly decrease with increasing social interactions. Although interactions between each child and the assistant were increased after the assistant's interaction in the interaction phases or a mere lapse of time, we observed very few interactions among the three participants. Contrary to our prediction, we obtained no significant correlation between subjective evaluation and group area for any test phase. The discrepancy between frequently observed social behaviours and low subjective evaluations seemed to result from instructions that required observers to evaluate three people's activity. It is possible that these instructions made it difficult for observers to evaluate group interactions, as the observable distances between each child and the assistant were almost the same in the test phases.

Our results conflicted with those of Tsuji et al. (2018), who found a decreasing tripartite group area in a free-play situation. Despite the similarities in the two studies' conditions in terms of number of children, interacting nonacquainted assistant, room size, and a free-play situation, they also had some differences. For instances, the participants used slightly different toys because these toys were tailored to their age and interest (Tsuji et al. used blocks, dinosaur dolls, animal dolls, suction toys, pictorial books, puzzles and so on). This might affect the frequency of showing behaviour and produce differential fluctuations in group area. Another case found that the discrepancy between the two studies may be due to participant characteristics, regarding children with ASD or typically developing children. In this study, although two typically developing children seldom approached the assistant, there was a relatively large number of interactions with the assistant through eye contact. That is, in the space of about four metres in this experiment, it was possible to communicate with the assistant through eye contact, verbal initiation and showing objects, while maintaining the same distance from the other person. However, these social behaviours are relatively challenging for children with ASD; therefore, one plausible explanation is that the children with ASD in the previous study approached others to interact, and their group area may have shown a different trend than the children in the present study, who had an extensive repertoire of social behaviours.

For activities that require less movement to interact with others, MCS measurements may not be stable due to several factors that affect approaching others. Additionally, it is known that children's interactions with peers or adults are influenced by activity settings (e.g. Booren et al., 2012). Therefore, in Experiment 2, we introduced a structured activity in which children could not fully play unless they were close to others, and examined the relationship between group area and social behaviours.

\section{Experiment 2}

\section{Participants}

Two typically developing boys participated in the experiment (Child 3: 6 years 6 months and Child 4: 6 years 0 months). The two boys attended the same kindergarten and had a good friendship. Two adult staff members (assistants) who did not participate in Experiment 1 joined in the group activity. The assistants were graduate students who majored in applied behaviour analysis and had not previously met the children. In the test phases, each assistant was assigned a different role during the experiment: reinforcement assistant (Assistant ${ }^{\mathrm{RF}}$ ) and non-reinforcement assistant (Assistant ${ }^{\text {Non-RF }}$ ). After the experiment, all participants were remunerated at a rate of approximately $\$ 10$ an hour.

\section{Apparatus and Setting}

Apparatus and setting were the same as in Experiment 1.

\section{Materials}

We provided a blown-up balloon for test phases and two types of toys (a bowling kit and a rubber ball) for interaction phases.

\section{Experimental Design}

Experiment 2 consisted of a single session including a test (test 1, test 2 and test 3 ) and an interaction phase. As in Experiment 1, MCS measurements and behavioural observation were performed during the test phases.

\section{Procedures}

Experiment 2 was carried out in the following order: test 1 , interaction 1 , interaction 2 , test 2 , interaction 3 , interaction 4 and test 3 (at the right side of Table 1). The introduction procedure and the experimenter's role in Experiment 2 were similar to that of Experiment 1. To encourage the children to interact with the assistants, the experimenter asked the children to toss a balloon to each assistant twice, after the children agreed to participate in the presented activities.

During the test phase, the children and assistants engaged in balloon volleyball, which required tossing a balloon towards each other and not letting the balloon touch the ground (actual scene is shown in the bottom-right of Fig. 1). Preliminary observations revealed that balloon volleyball was an attractive game for pre-schoolers and was available for typically developing children and children with ASD. Participants played balloon volleyball for four minutes after either child tossed a balloon towards the centre of the activity 
circle. For the children, they moved around freely and tossed the balloon within the activity circle. For the assistants, they remained at the edge of the activity circle and tossed a balloon within their reach. The distance between the two assistants was about $320 \mathrm{~cm}$, and there were spaces of about $40 \mathrm{~cm}$ between the assistants and the activity circle. The movable range of each assistant was indicated by colour tape on the floor. These procedures ensured that interactions between the children and assistants occurred only when the children approach the assistants. To reduce position bias, the assistants switched positions for each test phase. When the balloon went out of the activity circle, the experimenter returned it.

Before the beginning of test 1 , the children received the following instructions:

Let's play balloon volleyball. You two can play together or play with the assistants, but the assistants can't go outside the lines. So, if you want to play with them, go near them.

The assistants preliminarily received the following instructions:

When the children approach, join the game with a smile. You can toss the balloon if you can reach it, but you must stay in your default position. Please do not give any instructions or requirements for passing the balloon.

In this experiment, to clearly observe contrast in group area, we provided the two assistants with different roles and calculated group area, with respect to each assistant. We had planned to determine assistants' roles in the interaction phases based on the frequency each assistant tossed the balloon at during the test 1 phase. The assistant who tossed the balloon more in test 1 was supposed to be assigned the Assistant ${ }^{\text {Non-RF }}$ role in the interaction phases, and the other was supposed to be assigned the Assistant ${ }^{\mathrm{RF}}$ role. However, because the number of tosses was equal for both assistants, they were actually assigned to each role at random in the interaction phases. (The number of tosses identified as equal numbers here differs from the number of tosses to assistants in Results. The number of tosses counted for determining the roles of the assistants included the number of successive tosses by the same assistant after the assistants tossed the balloon).

During the interaction phases, there were two types of periods where either the Assistant ${ }^{\mathrm{RF}}$ or Assistant ${ }^{\mathrm{Non}-\mathrm{RF}}$ played with the two children. In interaction 1 and interaction 4 , Assistant ${ }^{\mathrm{RF}}$ played bowling or catch with the two children for four minutes. In interaction 2 and interaction 3, Assistant ${ }^{\text {Non-RF }}$ played bowling or catch with the two children for four minutes. While one assistant interacted with the children, the other assistant waited outside the experimental room. In each phase, Assistant ${ }^{\mathrm{RF}}$ intended to reinforce the children's communicative responses, by exhibiting positive affect such as smiling, laughing and using a playful vocal tone. Assistant ${ }^{\text {Non-RF }}$ stayed out of the child's play and watched with no expression.

\section{Measures and Dependent Variables}

Location Information with MCS During the test phase, as in Experiment 1, MCS continued real-time measurement and recording of spatial positions for the participants' and assistant's heads in 3D space. In Experiment 2, we calculated and analysed two tripartite group areas using MCS. One group area consisted of both children and Assistant ${ }^{\mathrm{RF}}$, and the other consisted of both children and Assistant ${ }^{\mathrm{Non}-\mathrm{RF}}$. After the experiment started, we dropped a marker on the floor to mark a countdown and analysed data collected for four minutes, starting from the time that the marker touched the floor. Unlike in the case of Experiment 1, there was no break.

Observational Measurement of Social Behaviours After the experiment, behavioural observation was executed by the first author using the video recorded for the three test phases. In Experiment 2, the activity chosen for the test phases was a balloon volleyball game, which required players to toss a balloon to each other. We focussed on whether the children would play with others, and we defined balloon tosses from one child to the other or to assistants as social behaviour (We attempted to use social behaviours typically measured in the related study, but we failed because of factors such as continuous smiling throughout the session and difficulty in distinguishing between eye contact and looking at the balloon.) 'To Assistant' was coded as an assistant tossing the balloon after either of the two children tossed it. 'To Peer' was coded as one child tossing the balloon, and then the other child tossing it. To reveal lack of social interactions, we also coded 'To Self' for each instance that the same child tossed the balloon more than once in a row. All tosses were coded using Noldus the Observer XT 14 software.

To evaluate the reliability of the observation, another independent observer scored all tosses across test phases for IOA. The first author and the independent observer counted the occurrences of the three types of tosses in relation to each child. As in Experiment 1, the total count IOA was calculated by dividing the number of smaller counts by the larger counts and then multiplying the result by 100 . For Child 3, the final IOA was $98.2 \%$ (To Assistant $=100.0 \%$ [38/38], To Peer $=$ 89.7\% [35/39] and To Self $=92.2 \%$ [83/90]). For Child 4, the final IOA was $95.2 \%$ (To Assistant $=100.0 \%$ [28/28], To Peer $=96.1 \%[49 / 51]$ and To Self $=75.0 \%[21 / 28])$.

Subjective Evaluation of Interaction After the experiment, four independent observers who did not know the purpose of the study watched a combined video on Observer XT 14 
software and evaluated the group interactions during each test phase, using a 5-point scale: $5=$ children are playing with adult 1 (Assistant ${ }^{\mathrm{RF}}$ ); $4=$ children are probably playing with adult 1 (Assistant $\left.{ }^{\mathrm{RF}}\right) ; 3=$ children are not playing with either adult; $2=$ children are probably playing with adult 2 (Assistant $^{\text {Non-RF }}$ ) and $1=$ children are playing with adult 1 (Assistant $^{\text {Non-RF}}$ ). The observers coded the duration of each score using the five assigned keys. The observations began with a score of ' 3 ', and all activities of participants in the videos were always evaluated with one of five scores. As in Experiment 1, these scores were mutually exclusive, and the observers could not code them at the same time. Before the evaluations, each observer practiced recording using an unrelated video. During the evaluation, the observers watched the recording at a normal speed without rewinding or pausing. The videos were watched in random order.

\section{Results}

Tripartite Group Area MCS measured $60 \mathrm{fps}$, and each test phase in Experiment 2 was four minutes; therefore, a total of 14,400 should have been obtained for group area. Valid group areas with Assistant ${ }^{\mathrm{RF}}$ were as follows: 13,177 (91.5\%) for test $1,12,732(88.4 \%)$ for test 2 and 12,136 (84.3\%) for test 3. Valid group areas with Assistant ${ }^{\text {Non-RF }}$ were as follows: $13,346(92.7 \%)$ for test $1,12,514(86.9 \%)$ for test 2 and $12,504(86.8 \%)$ for test 3 . As with Experiment 1, we calculated average group area every second and analysed a total of $240 \mathrm{~s}$ of data.

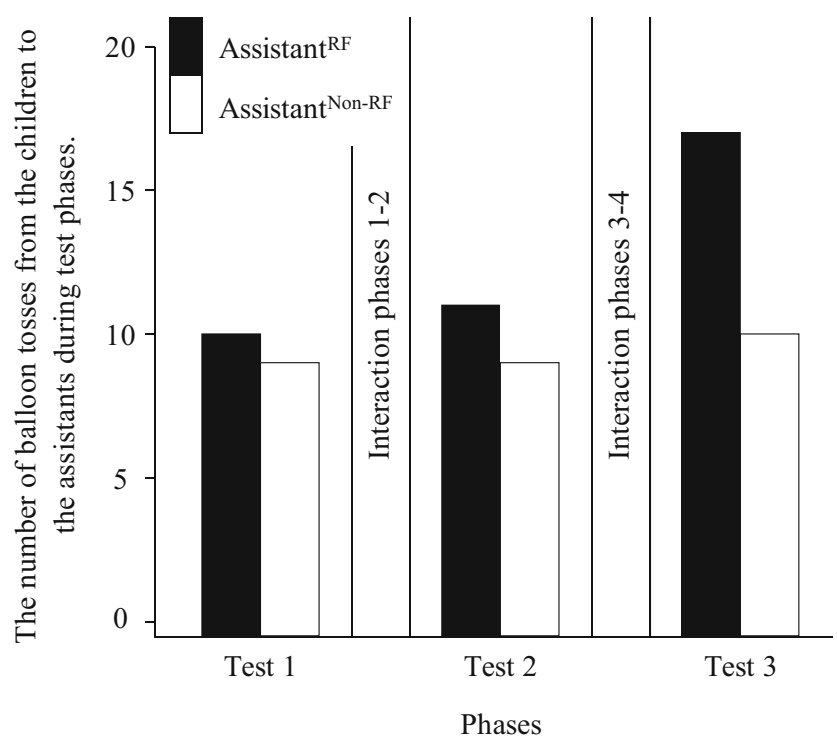

Fig. 4 The number of balloon tosses from both children to the assistants during test phases in Experiment 2. The black bars represent the assistant who exhibited positive affect and intended to reinforce the child's communicative responses in the interaction phases. The white bars represent the assistant who intended to provide no reinforcement in the interaction phases
Fluctuations of two group areas are shown by solid lines in Fig. 2. For Assistant ${ }^{\mathrm{RF}}$, the average group areas in each phase were $0.64 \mathrm{~m}^{2}(S D=0.450)$ for test $1,0.67 \mathrm{~m}^{2}(S D=0.564)$ for test 2 and $0.82 \mathrm{~m}^{2}(S D=0.560)$ for test 3 . For Assistant ${ }^{\text {Non-RF }}$, the average group areas in each phase were $0.55 \mathrm{~m}^{2}(S D=$ $0.389)$ for test $1,0.69 \mathrm{~m}^{2}(S D=0.503)$ for test 2 and $0.70 \mathrm{~m}^{2}$ $(S D=0.480)$ for test 3 . In this experiment, the values for group area were spread out over a wider range than those of Experiment 1. For both assistants, the average group areas increased as the session progressed.

Frequency of Social Behaviours Figure 4 shows the total frequency of balloon tosses for both children. For Assistant ${ }^{\mathrm{RF}}$, 'To Assistant' increased from test 1 to test 3 (test $1=10$, test $2=11$ and test $3=17$ ). For Assistant ${ }^{\text {Non-RF }}$, 'To Assistant' generally remained flat (test $1=9$, test $2=9$ and test $3=10$ ). 'To Peer' gradually decreased as the session progressed, whereas 'To Self' increased (test $1=38$, test $2=26$, test $3=$ 21 and test $1=23$, test $2=34$, test $3=42$, respectively).

Subjective Evaluation of Interaction To compare the results of Experiment 2 with those of Experiment 1, the four observers' subjective evaluation scores on a 5-point scale were translated into a scores on a 3-point scale for each assistant (for Assistant ${ }^{\mathrm{RF}}$, a score of ' 5 ' $=$ ' 3 ', ' 4 ' $=$ ' 2 ' ' and ' 3 ' $=$ ' 1 '; for Assistant $^{\text {Non-RF }}$, a score of ' 1 ' $=$ ' 3 ', ' 2 ' $=$ ' 2 ' and ' 3 ' $={ }^{\prime} 1$ ') . Then, they were averaged for every second. Transitions in average subjective evaluation is shown by the dashed lines in Fig. 2. For Assistant ${ }^{\mathrm{RF}}$, average subjective evaluations were $1.18(S D=0.352)$ for test $1,1.18(S D=0.378)$ for test 2 and $1.30(S D=0.529)$ for test 3 . For Assistant ${ }^{\text {Non-RF }}$, average subjective evaluations were $1.10(S D=0.266)$ for test $1,1.13$ $(S D=0.297)$ for test 2 and $1.21(S D=0.383)$ for test 3 . Although a score of ' 1 ' was still most common in each phase, contrary to Experiment 1, a score of ' $3=$ they are playing together' was observed in all phases.

Correspondence between Group Area and Subjective Evaluation Spearman's rank correlation coefficients between group area and subjective evaluation were calculated with respect to each phase. For Assistant ${ }^{\mathrm{RF}}$, we observed low negative relationships for test $1\left(r_{s}=-0.16, p=0.014\right)$ and for test $3\left(r_{s}=-0.25, p=0.000\right)$. For Assistant ${ }^{\text {Non-RF }}$, we observed low negative relationships for test $1\left(r_{s}=-0.19, p=0.003\right)$ and for test $3\left(r_{s}=-0.26, p=0.000\right)$. For test 2 , we observed no relationships between the two dependent variables for either Assistant ${ }^{\mathrm{RF}}$ or Assistant ${ }^{\mathrm{Non}-\mathrm{RF}}\left(r_{s}=-0.07, p=0.319\right.$ and $r_{s}=-0.11, p=0.098$, respectively).

Time-Window Sequential Analysis To investigate sequential associations among subjective evaluations, balloon tosses and group area, we implemented a time-window sequential analysis that tested whether a target event occurred within a 
certain temporal window, after an antecedent event occurred (Yoder \& Tapp, 2004). In this experiment, we used a brief time window of five seconds for all the analyses. Yule's $Q$ and adjusted $z$-scores were used for all analyses. Yule's $Q$ was calculated as a measure of sequential associations. This index ranges from -1.0 to 1.0 and can be interpreted similarly to a correlation coefficient. Positive Yule's $Q$ values mean the target event is more likely to occur within than outside the antecedent time window, negative Yule's $Q$ values mean that the occurrence of the target event within the antecedent time window is less than outside the antecedent time window, and zero values mean there is no association (Yoder et al., 2018). To test whether differences between observed and expected sequential frequency occurred by chance, we calculated $z$-scores (adjusted residual) by subtracting expected sequential frequency from observed sequential frequency and dividing the standard deviation of the difference (Yoder \& Tapp, 2004).

First, we examined the association between balloon tosses as the antecedent event and subjective evaluation as the target event. Three types of balloon tosses were coded every second, based on whether it did or did not occur. For subjective evaluations, scores of 2 or 3 in the original results were translated as occurrences in evaluations as the participants played together, and a score of 1 was translated as non-occurrences. As shown at the top of Table 2, for Assistant ${ }^{R F}$, changes in subjective evaluations significantly occurred within the time window of the antecedent tosses to the assistant (Yule's $Q$ scores [z-scores] for test $1=0.97$ [9.97], test $2=0.97$ [11.00] and test $3=0.99$ [13.51]). For Assistant ${ }^{\mathrm{Non}-\mathrm{RF}}$, similar results were obtained (Yule's $Q$ score [z-scores] for test $1=0.99$ [12.51], test $2=0.96[10.71]$ and test $3=0.93$ [9.43]).

Next, we examined the association between balloon tosses as the antecedent event and small group area as the target event. We defined small group area as group area below the 25th percentile of the total group area in each phase and coded whether or not it occurred every second. As shown in the middle of Table 2, small group area was more likely to occur within the time window of the antecedent tosses to the assistant, except for Assistant ${ }^{\mathrm{RF}}$ for test 1. Significant differences from chance were obtained for Assistant ${ }^{\mathrm{RF}}$ in test 3 (Yule's $Q$

Table 2 The results of time-window sequential analysis in Experiment 2

\begin{tabular}{|c|c|c|c|c|c|c|}
\hline & \multicolumn{2}{|l|}{ Test 1} & \multicolumn{2}{|l|}{ Test 2} & \multicolumn{2}{|l|}{ Test 3} \\
\hline & Yule's $Q$ & z-score & Yule's $Q$ & z-score & Yule's $Q$ & z-score \\
\hline \multicolumn{7}{|l|}{ Reinforcement assistant } \\
\hline To Assistant $\rightarrow$ Subjective evaluation & 0.97 & $9.97 * *$ & 0.97 & $11.00 * *$ & 0.99 & $13.51 * *$ \\
\hline To Peer $\rightarrow$ Subjective evaluation & -0.50 & $-3.62 * *$ & -0.59 & $-4.11 * *$ & -0.22 & -1.42 \\
\hline To Self $\rightarrow$ Subjective evaluation & 0.18 & 1.20 & -0.45 & $-2.90 * *$ & -0.17 & -1.13 \\
\hline \multicolumn{7}{|l|}{ Non-reinforcement assistant } \\
\hline To Assistant $\rightarrow$ Subjective evaluation & 0.99 & $12.51 * *$ & 0.96 & $10.71 * *$ & 0.93 & $9.43 * *$ \\
\hline To Peer $\rightarrow$ Subjective evaluation & -0.18 & -1.01 & -0.46 & $-2.94 * *$ & -0.01 & -0.07 \\
\hline To Self $\rightarrow$ Subjective evaluation & -0.16 & -0.87 & -0.20 & -1.19 & -0.28 & $-1.97 *$ \\
\hline \multicolumn{7}{|l|}{ Reinforcement assistant } \\
\hline To Assistant $\rightarrow$ Small group area & -0.28 & -1.20 & 0.07 & 0.39 & 0.50 & $3.24 * *$ \\
\hline To Peer $\rightarrow$ Small group area & 0.30 & 1.90 & -0.36 & $-2.36^{*}$ & -0.22 & -1.26 \\
\hline To Self $\rightarrow$ Small group area & -0.03 & -0.20 & 0.20 & 1.27 & -0.22 & -1.39 \\
\hline \multicolumn{7}{|l|}{ Non-Reinforcement assistant } \\
\hline To Assistant $\rightarrow$ Small group area & 0.36 & $2.05^{*}$ & 0.87 & 1.50 & 0.52 & $3.36^{* *}$ \\
\hline To Peer $\rightarrow$ Small group area & 0.09 & 0.59 & -0.18 & -1.15 & -0.03 & -0.20 \\
\hline To Peer $\rightarrow$ Small group area & 0.03 & 0.20 & 0.30 & 1.95 & -0.40 & -2.56 \\
\hline \multicolumn{7}{|l|}{ Reinforcement assistant } \\
\hline To Assistant $\rightarrow$ Large group area & -0.66 & $-2.74 * *$ & -0.40 & -1.84 & -0.81 & $-3.60 * *$ \\
\hline To Peer $\rightarrow$ Large group area & 0.12 & 0.78 & 0.38 & $2.50 *$ & 0.31 & $1.98 *$ \\
\hline To Self $\rightarrow$ Large group area & 0.10 & 0.63 & -0.22 & -1.40 & 0.06 & 0.37 \\
\hline \multicolumn{7}{|l|}{ Non-Reinforcement assistant } \\
\hline To Assistant $\rightarrow$ Large group area & -0.66 & $-2.74 * *$ & -0.40 & -1.84 & -0.81 & $-3.60 * *$ \\
\hline To Peer $\rightarrow$ Large group area & -0.02 & -0.16 & -0.03 & -0.20 & 0.13 & 0.81 \\
\hline To Self $\rightarrow$ Large group area & -0.15 & -0.95 & 0.50 & $-3.13 * *$ & 0.66 & $4.69 * *$ \\
\hline
\end{tabular}

Arrows direction indicate the time sequence; antecedent events $\rightarrow$ target events. $* p<0.05$, $* * p<0.01$; two-tailed 
$[z$-scores $]=0.50$ [3.24]), for Assistant ${ }^{\text {Non-RF }}$ in test 1 (Yule's $Q[z$-scores $]=0.36$ [2.05]) and for Assistant ${ }^{\text {Non-RF }}$ in test 3 (Yule's $Q$ [z-scores] $=0.52$ [3.36]). For Assistant ${ }^{\mathrm{RF}}$, a negative Yule's $Q$ score was obtained in the association between tosses to the assistant and small group area; however, it did not reach significance.

Finally, we examined the association between balloon tosses as the antecedent event and large group area as the target event (at the bottom of Table 2). We defined large group area as group area above the 75 th percentile of the total group area in each phase and coded whether it occurred or not every second. For Assistant ${ }^{\mathrm{RF}}$, large group area was less likely to occur within the time window as the antecedent tosses to the assistant (Yule's $Q$ score [z-scores] for test $1=-0.62$ [-2.52], test $3=-0.62[-3.08])$. For Assistant ${ }^{\text {Non-RF }}$, similar results were obtained (Yule's $Q$ score $[z$-scores] for test $1=-0.66$ $[-2.74]$, test $3=-0.81[-3.60])$. We observed significant differences from chance in all associations between tosses to an assistant and large group area, except for in test 2 for both assistants.

Supplementary Episodes Overall, the two children kept moving inside the activity circle during test phases. However, in test 2 , the visible distance between participants sometimes did not vary, because Child 1 fell on the floor twice. At test 1 , the children were often close enough to touch shoulders; thus, the positions of each participant were sometimes in a line.

\section{Discussion}

In contrast to Experiment 1, distances between the children and assistants visibly changed in Experiment 2. We also confirmed that the independent observers rated the four participants in Experiment 2 as playing together. However, although we attempted to obtain contrasting group areas by assigning different roles to the two assistants, this did not work as planned. In test 3, the average group area of Assistant ${ }^{\mathrm{RF}}$, who played with the children more frequently, was larger than that of Assistant ${ }^{\text {Non-RF. }}$. When group areas were summarised as a representative value, the relationship with the other indices became unclear.

Correlation analysis results for subjective evaluation and group area suggested that the more group members played together, the smaller group area was likely to be. In test 2 , however, there was no correlation between tosses to Assistant ${ }^{\text {Non-RF }}$ and group area. One child fell on the floor twice during test 2 , and the observable group area that followed the assistants' balloon tossing varied within a relatively wide range. This may have resulted in the low correlation between subjective evaluation and tosses to Assistant ${ }^{\text {Non-RF }}$ in test 2.

When we visualised subjective evaluation and group area as time sequential data, both measures seemed to change in conjunction with balloon tosses to assistants. Time-window sequential analysis revealed that subjective evaluation was strongly associated with balloon tosses to the two assistants. Furthermore, additional analyses for the association between balloon tosses and group area size suggested that when participants played together, group area was more likely to be small (below $25 \%$ of the total group area), or less likely to be large (above $75 \%$ of the total group area). Taking account of negative correlations between subjective evaluation and group area, it would be possible to determine the occurrence of social interactions within a group, to some extent, by visualising and examining the time sequential transition of the data. However, because the strength of achieved correlations between subjective evaluation and group area were, in total, lower than Yule's $Q$, which indicated the associations between subjective evaluation and balloon tosses, the behavioural data (balloon tosses) may have reflected social interactions more than group area. Furthermore, since we partially obtained statistically significant occurrences and nonoccurrences in the time-window sequential analysis, using group area as a measure of social interactions should be considered limited in the present study.

\section{General Discussion}

In this study, we conceptually replicated an experiment by Tsuji et al. (2018) that reported the measurability of group area using MCS and investigated whether group area could be used as a measurement of pre-school children's social interactions. As with the previous study, we succeeded in quantitatively measuring individuals' positions during activities and calculated group area. Our results suggested that the index of group area is not stable in activities in which it is difficult to predict how people will behave, but can be used as a relatively reliable index of social interactions in activities where behaviours are predictable, to some extent.

In Experiment 2, although the children engaged in increased levels of interaction with the assistant who intended to reinforce the child's communicative responses during the interaction phases, average group area also increased as child-to-assistant interactions increased. In contrast, the time-window sequential analysis suggested that group area immediately after the interactions was relatively small, rather than large. Furthermore, regarding associations between balloon tosses to the assistants and large group area, all Yule's $Q$ values were in the same negative direction and were more consistent than in analysis of small group area. These results suggested that a reliable assessment would be obtained if we estimated the occurrences of social interactions within the group, based on a larger group area rather than a small group area. Thus, group area may be used as one index to quantitatively identify a pre-schooler's group which has few interactions and is needed for some interventions.

Stronger associations between subjective evaluation and behavioural data than between subjective evaluation and group area 
indicated that direct observation of individual behaviour can provide a more valid and reliable assessment of social interactions within a group. However, implementing direct observation of behaviour requires observer training to record occurrences of precisely defined target behaviours, which often requires much time and effort (e.g. Yoder et al., 2018). Although our method did not produce as much behavioural data, it was found that group area showed a relationship with social interaction. When providing behavioural support to children (e.g. children with ASD who have difficulties with social interactions), it may be worth considering using group area, which can be computed automatically, as a preliminary screening index before conducting an assessment based on existing direct and indirect observation.

However, when interpreting the present study's findings, we should keep in mind that, for typically developing children, group area is a measurement that depends on activities and setting. We found no relationship between group area and other measures in the activity where the participant could move, but did not need to (i.e. free play), or in the activity in which one participant's movements were restricted, due to falling on the floor. These results indicated that estimating social interactions using group area is difficult in a situation in which individuals rarely approach each other. At this point, even if it were possible to screen for low-interactive populations using group area, this would be done in extremely limited conditions.

Further studies must be conducted across activities and participants to consider how group area can be applied to practical environments such as schools. Regarding the factor of participants, if another child had participated instead of an assistant (i.e. a group of three children), we could have observed increasing peer interactions within a small group area, even during free play (Booren et al., 2012). Moreover, future research may need to consider gender differences as well because the two experiments in this study that yielded different results differed in the gender of the participants (In this study, we considered that all children were 5-6 years old and could engage in social play, regardless of gender differences [e.g. Barbu et al., 2011] and did not discuss the influence of gender). It will be necessary to determine by systematic replications under which conditions group area is a valid measure considering various factors such as activities and characteristics of participants.

We initially predicted that if more than three participants interacted with shorter interpersonal distances between them, tripartite group area would be smaller. Group area, however, can be small even when individuals are in a row. Thus, tripartite group area can be small when one person is standing still while the other two are interacting with each other at a distance. This problem could be common to all phases, and the reason for the overall low correlation may be due to this aspect of group area, particularly regarding test 1 for Experiment 2, in which a different result was obtained by time-window sequential analysis. In order to more accurately measure group social interactions using MCS, future studies would be necessary to adjust group area with a different index. For example, body and head orientation could also be calculated using MCS (Kita et al., 2017), and determining whether group members are facing each other may reduce the influence of incidentally obtained group area.

In this study, we calculated group area using OptiTrack Flex 3 MCS; however, MCS is not necessarily required to measure group area. If another device which measured individuals' positions was available, group area could be calculated. For example, Microsoft Kinect, a relatively low-cost device, has competitive motion tracking performance to OptiTrack V100:R2 (Flex 3 before rebranded) in a certain condition (Chang et al., 2012; Dubois \& Bresciani, 2018). Another technique could be to estimate individuals' relative positions based on radio field intensities (Messinger et al., 2019; Miura et al., 2013; Yamada et al., 2006). Although high-fidelity devices, such as OptiTrack, would be appropriate to examine the validity and reliability of group area, the use of alternative tools should also be considered for the dissemination of this index in applied and clinical fields.

\section{Conclusion}

In this pilot study, we used the MCS to assess social interactions in a three-person group that involves typically developing children. Our results showed that the tripartite group area calculated via the MCS had the potential for assessing social interactions. Although the validity and reliability of group area as a social interaction index were not extended to behavioural data obtained via direct observation, our technology-based approach allows for an evaluation of real-time interaction between more than three people in an ecologically valid way. Nevertheless, this pilot study has shown that group area measured using the MCS can serve as a social interaction index in an extremely limited environment that requires movement when interacting with others. For the practical use of group area in daily and clinical settings, it will be necessary to determine the systematic replication conditions under which group area becomes an effective index, considering such factors as activities and participants' characteristics.

Acknowledgments This study was supported by JST CREST Grant Number JPMJCR14E2, Japan. We would like to thank Maiko Akimoto, Masayo Koyama, and Hiroki Sato for their invaluable assistance in conducting the experiments. We are grateful to Ayane Suzuki for assistance in conducting reliability check and data analyses, to Yaqiang Wei for helpful comments on English writing.

Author Contributions Masashi Tsukamoto and Airi Tsuji should be considered joint first author. Masashi Tsukamoto, Airi Tsuji and Satoru Sekine designed the research. Masashi Tsukamoto and Satoru Sekine conducted the experiments. Airi Tsuji and Kenji Suzuki analysed engineering data. Masashi Tsukamoto, Takahide Omori and Junichi Yamamoto analysed overall data. Masashi Tsukamoto wrote the first 
manuscript. All authors contributed to final manuscript and approved to submit it.

\section{Declarations}

This study was supported by JST CREST Grant Number JPMJCR14E2 and JSPS KAKENHI Grant Number JP20K14071, Japan.

The authors have no conflict of interest to declare.

Keio University's Research Ethics Committee approved this study. Before starting, parents of the participating children received verbal and written explanations about this study and provided written informed consent for participation and publication.

The data that support the findings of this study are available on request from the corresponding author. The data are not publicly available due to privacy or ethical restrictions.

Open Access This article is licensed under a Creative Commons Attribution 4.0 International License, which permits use, sharing, adaptation, distribution and reproduction in any medium or format, as long as you give appropriate credit to the original author(s) and the source, provide a link to the Creative Commons licence, and indicate if changes were made. The images or other third party material in this article are included in the article's Creative Commons licence, unless indicated otherwise in a credit line to the material. If material is not included in the article's Creative Commons licence and your intended use is not permitted by statutory regulation or exceeds the permitted use, you will need to obtain permission directly from the copyright holder. To view a copy of this licence, visit http://creativecommons.org/licenses/by/4.0/.

\section{References}

American Psychiatric Association. (2013). Diagnostic and statistical manual of mental disorders, $5^{\text {th }}$ edition (DSM-5). American Psychiatric Pub.

Bakeman, R., \& Adamson, L. B. (1984). Coordinating attention to people and objects in mother-infant and peer-infant interaction. Child Development, 55(4), 1278-1289. https://doi.org/10.2307/1129997.

Barbu, S., Cabanes, G., \& Le Maner-Idrissi, G. (2011). Boys and girls on the playground: Sex differences in social development are not stable across early childhood. PLoS One, 6(1), e16407. https://doi.org/10. 1371/journal.pone.0016407.

Booren, L. M., Downer, J. T., \& Vitiello, V. E. (2012). Observations of children's interactions with teachers, peers, and tasks across preschool classroom activity settings. Early Education and Development, 23(4), 517-538. https://doi.org/10.1080/10409289. 2010.548767.

Brassard, M. R., \& Boehm, A. E. (2007). Preschool assessment: Principles and practices. Guilford Press.

Candini, M., Giuberti, V., Manattini, A., Grittani, S., di Pellegrino, G., \& Frassinetti, F. (2017). Personal space regulation in childhood autism: Effects of social interaction and person's perspective. Autism Research, 10(1), 144-154. https://doi.org/10.1002/aur.1637.

Chang, C. Y., Lange, B., Zhang, M., Koenig, S., Requejo, P., Somboon, N.,. . and Rizzo, A. A. (2012). Towards pervasive physical rehabilitation using Microsoft Kinect. Retrieved from https://ieeexplore. ieee.org/Xplore/home.jsp. In 6th international conference on pervasive computing technologies for healthcare (PervasiveHealth) and workshops, 159-162.

Dubois, A., \& Bresciani, J. P. (2018). Validation of an ambient system for the measurement of gait parameters. Journal of Biomechanics, 69 , 175-180. https://doi.org/10.1016/j.jbiomech.2018.01.024.
Fantuzzo, J., Coolahan, K., Mendez, J., McDermott, P., \& Sutton-Smith, B. (1998a). Contextually relevant validation of peer play constructs with African American head start children: Penn interactive peer play scale. Early Childhood Research Quarterly, 13(3), 411-431. https://doi.org/10.1016/S0885-2006(99)80048-9.

Fantuzzo, J., Mendez, J., \& Tighe, E. (1998b). Parental assessment of peer play: Development and validation of the parent version of the Penn interactive peer play scale. Early Childhood Research Quarterly, 13(4), 659-676. https://doi.org/10.1016/S08852006(99)80066-0.

Hayduk, L. (1983). Personal space: Where we now stand. Psychological Bulletin, 94(2), 293-335. https://doi.org/10.1037/0033-2909.94.2. 293.

Howes, C., \& Matheson, C. C. (1992). Sequences in the development of competent play with peers: Social and social pretend play. Developmental Psychology, 28(5), 961-974. https://doi.org/10. 1037/0012-1649.28.5.961.

Kazdin, A. E. (1979). Unobtrusive measures in behavioral assessment. Journal of Applied Behavior Analysis, 12(4), 713-724. https://doi. org/10.1901/jaba.1979.12-713.

Kennedy, D. P., \& Adolphs, R. (2014). Violations of personal space by individuals with autism spectrum disorder. PLoS One, 9(8), e103369. https://doi.org/10.1371/journal.pone.0103369.

Kita, Y., Gunji, A., Sakuma, R., Goto, T., Okumura, Y., \& Inagaki, M. (2017). Training attention to the other's need improves helping behaviors in children with autism spectrum disorder: A behavioral analysis using a two-dimensional motion capture system. Frontiers in Education, 2, 1-7. https://doi.org/10.3389/feduc.2017. 00050 .

Lord, C., Rutter, M., DiLavore, P., Risi, S., Gotham, K., \& Bishop, S. (2012). Autism diagnostic observation schedule-2nd edition (ADOS-2). Western Psychological Corporation.

Messinger, D. S., Prince, E. B., Zheng, M., Martin, K., Mitsven, S. G., Huang, S., Stölzel, T., Johnson, N., Rudolph, U., Perry, L. K., Laursen, B., \& Song, C. (2019). Continuous measurement of dynamic classroom social interactions. International Journal of Behavioral Development, 43(3), 263-270. https://doi.org/10.1177/ 0165025418820708.

Miltenberger, R.G. (2017). Behavior modification: Principles and procedures (6th ed). Cengage Learning.

Miura, A., Isezaki, T., \& Suzuki, K. (2013). Social playware with an enhanced reach for facilitating group interaction. In $\mathrm{CHI}^{\prime} 13$ extended abstracts on human factors in computing systems (pp. 11551160). doi:https://doi.org/10.1145/2468356.2468562.

Mundy, P., \& Gomes, A. (1998). Individual differences in joint attention skill development in the second year. Infant Behavior and Development, 21(3), 469-482. https://doi.org/10.1016/S01636383(98)90020-0.

Neisworth, J. T., \& Bagnato, S. J. (2004). The mismeasure of young children: The authentic assessment alternative. Infants and Young Children, 17(3), 198-212. Retrieved from https://journals.lww.com/ iycjournal/pages/default.aspx. https://doi.org/10.1097/00001163200407000-00002.

Thompson, T., Felce, D., \& Symons, F. J. (2000). Behavioral observation: Technology and applications in developmental disabilities. Paul H. Brookes.

Tsuji, A., Enomoto, T., Matsuda, S., Yamamoto, J., \& Suzuki, K. (2018). Modeling and quantitative measurement method of the tripartite interpersonal distance dynamics for children with ASD. In International conference on computers helping people with special needs, 523-526. https://doi.org/10.1007/978-3-319-94277-3_81.

Tsuji, A., Matsuda, S., \& Suzuki, K. (2016). Interpersonal distance and face-to-face behavior during therapeutic activities for children with ASD. In International Conference on Computers Helping People with Special Needs, 367-374. https://doi.org/10.1007/978-3-31941267-2 52 . 
Vernon, T. W., Koegel, R. L., Dauterman, H., \& Stolen, K. (2012). An early social engagement intervention for young children with autism and their parents. Journal of Autism and Developmental Disorders, 42(12), 2702-2717. https://doi.org/10.1007/s10803-012-1535-7.

Yamada, S., Takayama, J. Y., \& Ohyama, S. (2006). Sensor node localization based on inequalities of radio field intensity-comparison of simulation and experiments. In SICE-ICASE International Joint Conference, 1947-52. https://doi.org/10.1109/SICE.2006.315350.

Yoder, P. J., Lloyd, B. P., \& Symons, F. J. (2018). Observational measurement of behavior (2nd ed.). Paul H. Brookes.
Yoder, P. J., \& Tapp, J. (2004). Empirical guidance for time-window sequential analysis of single cases. Journal of Behavioral Education, 13(4), 227-246. https://doi.org/10.1023/B:JOBE. 0000044733.03220.a9.

Publisher's Note Springer Nature remains neutral with regard to jurisdictional claims in published maps and institutional affiliations. 